\title{
Low-mass Higgs decays to four leptons at one loop and beyond
}

\author{
Bernd A. Kniehl and Oleg L. Veretin \\ II. Institut für Theoretische Physik, Universität Hamburg, Luruper Chaussee 149, 22761 Hamburg, Germany
}

(Received 29 June 2012; published 13 September 2012)

\begin{abstract}
The ongoing searches for Higgs-boson signals in data taken at the CERN LHC and the Fermilab Tevatron crucially rely on the decay channels $H \rightarrow Z \ell \ell$ and $H \rightarrow W \ell \nu_{l}$. We present a precision study of the partial widths of these decay channels including the full one-loop electroweak corrections and the dominant contributions at two and three loops, of $\mathcal{O}\left(G_{F}^{2} m_{t}^{4}\right), \mathcal{O}\left(G_{F} m_{t}^{2} \alpha_{s}\right)$, and $\mathcal{O}\left(G_{F} m_{t}^{2} \alpha_{s}^{2}\right)$. Since the invariant mass of the off-shell intermediate boson is relatively low in the mass window $115 \mathrm{GeV}<m_{H}<129 \mathrm{GeV}$ of current interest, lepton mass effects are relevant, especially for the $\tau$ lepton.
\end{abstract}

DOI: 10.1103/PhysRevD.86.053007

PACS numbers: 14.80.Bn, 12.15.Lk, 12.38.Bx, 13.40.Ks

\section{INTRODUCTION}

The ATLAS and CMS collaborations at the $p p$ collider LHC are presently closing in on the Higgs boson of the standard model (SM). The combined ATLAS results, based on the full data set of up to $4.9 \mathrm{fb}^{-1}$ recorded during the 2011 operation at the center-of-mass energy $\sqrt{s}=7 \mathrm{TeV}$, excluded the Higgs-boson mass $\left(m_{H}\right)$ windows from 110.0 to $117.5 \mathrm{GeV}$, from 118.5 to $122.5 \mathrm{GeV}$, and from 129 to $539 \mathrm{GeV}$ at the $95 \%$ confidence level (C.L.) [1]. A small excess of signal events over the estimated background was observed around $m_{H}=$ $126 \mathrm{GeV}$ with a local significance of 2.5 standard deviations $(\sigma)$. The CMS Collaboration excluded the $m_{H}$ window from 127.5 to $600 \mathrm{GeV}$ at $95 \%$ C.L. exploiting approximately $5 \mathrm{fb}^{-1}$ of data at $\sqrt{s}=7 \mathrm{TeV}$ [2]. They observed some excess in the $m_{H}$ window from 115 to $128 \mathrm{GeV}$, with a maximum local significance of $2.8 \sigma$ at $m_{H}=125 \mathrm{GeV}$.

The CDF and D0 collaborations at the $p \bar{p}$ collider Tevatron jointly excluded the $m_{H}$ windows from 100 to $106 \mathrm{GeV}$ and from 141 to $184 \mathrm{GeV}$ at $95 \%$ C.L. by analyzing a luminosity of up to $10 \mathrm{fb}^{-1}$ collected at $\sqrt{\mathrm{s}}=$ $1.96 \mathrm{TeV}$ [3]. They observed an excess of signal over background in the $m_{H}$ window from 115 to $135 \mathrm{GeV}$, with a local significance of $2.2 \sigma$ at $m_{H}=120 \mathrm{GeV}$. The ALEPH, DELPHI, L3, and OPAL collaborations at the CERN $e^{+} e^{-}$collider LEP established a $m_{H}$ lower bound of $114.4 \mathrm{GeV}$ at $95 \%$ C.L. using a total of $2.461 \mathrm{fb}^{-1}$ data collected at $189 \mathrm{GeV}<\sqrt{s}<209 \mathrm{GeV}$ [4].

The searches at the LHC for the SM Higgs boson in the low- $m_{H}$ region mainly rely on its decay channels $H \rightarrow \gamma \gamma$ $[5,6], \quad H \rightarrow Z Z^{*} \rightarrow \ell^{+} \ell^{-} \ell^{\prime+} \ell^{\prime-} \quad[7,8], \quad H \rightarrow W^{+} W^{-*}$, $W^{+*} W^{-} \rightarrow \ell^{+} \nu_{\ell} \ell^{\prime-} \bar{\nu}_{\ell^{\prime}}[9,10]$, where off-mass-shell particles are marked by asterisks and $\ell, \ell^{\prime}=e, \mu$. Decay channels of lesser significance include $H \rightarrow \tau^{+} \tau^{-}$ $[11,12]$ and, in $W^{ \pm} H$ and $Z H$ associated production with subsequent $W^{ \pm} \rightarrow \ell^{ \pm} \stackrel{(-)}{\nu} \ell$ and $Z \rightarrow \ell^{+} \ell^{-}$decays, $H \rightarrow b \bar{b}$ $[13,14]$. In the following, we briefly review the state of the art regarding the theoretical predictions for the respective partial decay widths. Comprehensive reviews of our theoretical knowledge of the SM Higgs boson may be found in Refs. $[15,16]$ and references cited therein.

As for the partial decay width $\Gamma(H \rightarrow \gamma \gamma)$, the lowestorder result was first obtained in Ref. [17]. The two-loop $\mathcal{O}\left(\alpha_{s}\right)$ [18] and three-loop $\mathcal{O}\left(\alpha_{s}^{2}\right)$ [19] QCD corrections are available. As for the two-loop $\mathcal{O}(\alpha)$ correction, the contributions induced by light [20] and heavy fermions [21,22] as well as the residual ones [23] are known.

As for the partial decay width $\Gamma(H \rightarrow b \bar{b})$, the one-loop $\mathcal{O}\left(\alpha_{s}\right)$ [24] and $\mathcal{O}(\alpha)[25,26]$ corrections have been known for a long time. As for the two-loop $\mathcal{O}\left(\alpha_{s}^{2}\right)$ correction, the leading [27] and next-to-leading [28] terms of the expansion in $m_{b}^{2} / m_{H}^{2}$ of the diagrams without top quarks are known. The diagrams containing a top quark can be divided into two classes: diagrams containing gluon selfenergy insertions, which are exactly known [29], and double-triangle diagrams, for which the four leading terms of the expansion in $m_{H}^{2} / m_{t}^{2}$ are known [30]. The $m_{b}$-independent terms of the two-loop $\mathcal{O}\left(\alpha^{2}\right)$ and $\mathcal{O}\left(\alpha \alpha_{s}\right)$ corrections may be obtained from the analogous $\mathcal{O}\left(\alpha_{s}^{2}\right)$ one [27] by adjusting coupling constants and color factors [31]. As for the two-loop correction of order $\mathcal{O}\left(x_{t} \alpha_{s}\right)$, where $x_{t}=G_{F} m_{t}^{2} /\left(8 \pi^{2} \sqrt{2}\right)$ parametrizes leading power corrections of top-quark origin, the universal part, which appears for any Higgs-boson decay to a fermion pair, was extracted from the full $\mathcal{O}\left(\alpha \alpha_{s}\right)$ result [32] and the nonuniversal one, which arises because bottom is the isopartner of top, using a low-energy theorem [33]. As for the two-loop $\mathcal{O}\left(x_{t}^{2}\right)$ correction, the universal [21,34] and nonuniversal [34] parts are both available. The $\mathcal{O}\left(G_{F}^{2} m_{H}^{4}\right)$ term [35], which is universal, dominates the electroweak two-loop correction in the large- $m_{H}$ regime. The three-loop $\mathcal{O}\left(\alpha_{s}^{3}\right)$ correction without top-quark contribution was calculated in the massless limit [36]. The correction induced by the top quark was subsequently found using an appropriate effective field theory [37]. As for the three-loop $\mathcal{O}\left(x_{t} \alpha_{s}^{2}\right)$ correction, the universal [38] and nonuniversal [39] parts are both known. The four-loop $\mathcal{O}\left(\alpha_{s}^{4}\right)$ correction without topquark contribution was calculated in the massless limit [40]. The residual theoretical uncertainty in the prediction of $\Gamma(H \rightarrow b \bar{b})$ was assessed in Ref. [41]. 
As for the partial decay width $\Gamma\left(H \rightarrow \tau^{+} \tau^{-}\right)$, we know the full one-loop $\mathcal{O}(\alpha)$ [25,26] and two-loop $\mathcal{O}\left(\alpha \alpha_{s}\right)$ [32] corrections as well as the dominant terms of $\mathcal{O}\left(x_{t}^{2}\right)$ [21] and $\mathcal{O}\left(G_{F}^{2} m_{H}^{4}\right)$ [35] at two loops, and the one of $\mathcal{O}\left(x_{t} \alpha_{s}^{2}\right)$ [38] at three loops.

The decays of the Higgs boson to two pairs of light fermions proceed almost exclusively via $W^{+(*)} W^{-(*)}$ or $Z^{(*)} Z^{(*)}$ pairs, the contributions involving the Yukawa couplings of the produced fermions being greatly suppressed, as will be explicitly demonstrated for $H \rightarrow Z \tau^{+} \tau^{-}$and $H \rightarrow W^{+} \tau^{-} \bar{\nu}_{\tau}$ in Sec. III. If the value of $m_{H}$ is large enough to allow for one or both intermediate bosons to be on mass shell, then such kinematic configurations will be extremely favored due to the resonating propagators [42]. In this case, it is natural to employ the narrow-width approximation, which implies that the resonating intermediate bosons are treated as real particles and their subsequent decays are accounted for by multiplication with the appropriate branching fractions. Such a procedure is also routinely employed when the cross section of a complete scattering process involving the unstable Higgs boson is factorized into the cross section of the Higgs-boson production mechanism and the branching fraction of the Higgs-boson decay channel. In the case of Higgs-boson decays to two light-fermion pairs, this has the advantage that the branching fractions $B(Z \rightarrow f \bar{f})$ and $B\left(W^{ \pm} \rightarrow f \bar{f}^{\prime}\right)$ may be taken to be the experimentally measured values, which naturally contain all radiative corrections. As for the partial decay widths $\Gamma\left(H \rightarrow W^{+} W^{-}\right)$and $\Gamma(H \rightarrow Z Z)$, which presuppose that $m_{H}>2 m_{V}$ with $V=W, Z$, the full one-loop $\mathcal{O}(\alpha)$ corrections [25,43-45], also including the subsequent decays into massless-fermion pairs for offshell $V$ bosons [46], the dominant two-loop terms of $\mathcal{O}\left(x_{t} \alpha_{s}\right)$ [47,48], $\mathcal{O}\left(x_{t}^{2}\right)$ [21], and $\mathcal{O}\left(G_{F}^{2} m_{H}^{4}\right)$ [49], and the three-loop term of $\mathcal{O}\left(x_{t} \alpha_{s}^{2}\right)$ [38] are available. In Ref. [46], the $\mathcal{O}\left(G_{F}^{2} m_{H}^{4}\right)$ corrections [49], which are only relevant for $m_{H} \gg 2 m_{V}$, and certain higher-order effects due to photonic final-state radiation off charged leptons, which are not logarithmically enhanced for the integrated partial decay widths, were also included.

The purpose of this paper is to present a precision study of the partial decay widths $\Gamma\left(H \rightarrow W^{ \pm} f \bar{f}^{\prime}\right)$ and $\Gamma(H \rightarrow Z f \bar{f})$, appropriate for the mass window $m_{V}<$ $m_{H}<2 m_{V}$ of topical interest, retaining the masses of the produced fermions and including the full one-loop corrections and the dominant higher-order terms. In the case of $H \rightarrow Z f \bar{f}$ with massless final-state fermions, the one-loop weak correction may be obtained [15] by crossing symmetry from the corresponding analysis of $e^{+} e^{-} \rightarrow Z H$ [50,51]. Here, we redo this calculation for massive finalstate fermions and incorporate the $\mathcal{O}\left(x_{t}^{2}\right), \mathcal{O}\left(x_{t} \alpha_{s}\right)$, and $\mathcal{O}\left(x_{t} \alpha_{s}^{2}\right)$ terms. We also perform the analogous analysis for $H \rightarrow W^{ \pm} f \bar{f}^{\prime}$. In fact, the numerical analyses for $H \rightarrow$ $Z \tau^{+} \tau^{-}$and $H \rightarrow W^{+} \tau^{-} \bar{\nu}_{\tau}$ in Sec. III will reveal that the finite $\tau$-lepton mass effects may exceed the radiative corrections in size in the low- $m_{H}$ window that is not excluded experimentally. Both channels are bound to be exploited for the search of a low- $m_{H}$ Higgs boson and/or the study of its properties in the long run. The $H \rightarrow \ell^{+} \ell^{-} \tau^{+} \tau^{-}$ channel is already being used by the CMS Collaboration in the high- $m_{H}$ range [52].

This paper is organized as follows: In Sec. II, we expose the structure of our analytical results and list an appropriate selection of our formulas. In Sec. III, we present our numerical analysis for the $H \rightarrow Z \ell^{+} \ell^{-}$and $H \rightarrow W^{+} \ell^{-} \bar{\nu}_{\ell}$ decay channels, which are relevant for the ongoing searches for Higgs-boson signals in data taken at the LHC and the Tevatron. In Sec. IV, we summarize our conclusions.

\section{ANALYTIC RESULTS}

We now present our analytic results. We work in the on-mass-shell renormalization scheme implemented with Fermi's constant $G_{F}$, instead of Sommerfeld's finestructure constant $\alpha$, and use the shorthand notations $h=m_{H}^{2}, w=m_{W}^{2}, z=m_{Z}^{2}$, and $c_{w}^{2}=1-s_{w}^{2}=w / z$.

\section{A. $H \rightarrow Z f \bar{f}$ decay}

We first consider the decay process $H \rightarrow Z f \bar{f}$ for a generic fermion $f$ of mass $m$, electric charge $Q$, and color multiplicity $N$, which is $N=1$ for leptons and $N=3$ for quarks. The $Z f \bar{f}$ vector and axial-vector couplings are proportional to $V=2 I-4 s_{w}^{2} Q$ and $A=2 I$, where $I= \pm 1 / 2$ is the third component of weak isospin of the left-handed component of $f$. Formally, the partial decay width of $H \rightarrow Z f \bar{f}$ and the one of $Z \rightarrow H f \bar{f}$, the radiative corrections to which were studied in Ref. [53], are related as [15]

$$
\Gamma_{H \rightarrow Z f \bar{f}}=-3\left(\frac{z}{h}\right)^{3 / 2} \Gamma_{Z \rightarrow H f \bar{f}},
$$

where the minus sign ensures that the phase space remains positive upon the interchange $z \leftrightarrow h$, and the factors 3 , $\sqrt{z / h}$, and $z / h$ adjust the spin average, flux, and phase space, respectively. In contrast to Ref. [53], we include here also the finite- $m$ corrections.

The distribution of the partial decay width $\Gamma_{H \rightarrow Z f f}$ in the $f \bar{f}$ invariant mass square $s=q^{2}$ may be written as

$$
\begin{aligned}
\frac{d \Gamma_{H \rightarrow Z f \bar{f}}}{d s}= & \frac{d \Gamma_{H \rightarrow Z f \bar{f}}^{0}}{d s}\left[\delta_{0}+\frac{3}{4}\left(\frac{\alpha}{\pi} Q^{2}+\frac{\alpha_{s}}{\pi} C_{F}\right) \delta_{1}\right. \\
& \left.+\delta_{\mathrm{w}}-\delta_{x_{t}}+\delta_{\text {res }}\right] \delta_{t},
\end{aligned}
$$

where

$$
\frac{d \Gamma_{H \rightarrow Z f \bar{f}}^{0}}{d s}=\frac{G_{F}^{2} z^{3}}{128 \pi^{3} h^{3 / 2}} N\left(V^{2}+A^{2}\right) \frac{C_{1}}{(s-z)^{2}},
$$

with $C_{1}=\sqrt{\lambda}[4 s+\lambda /(3 z)][50]$ and $\lambda=s^{2}+z^{2}+h^{2}-$ $2(s z+z h+h s)$, is the tree-level result for $m=0[54,55]$, 
the factor $\delta_{0}$ restores the full $m$ dependence of the latter, $C_{F}=\left(N^{2}-1\right) /(2 N), \delta_{1}$ is the coefficient shared by the $\mathcal{O}(\alpha)$ QED and $\mathcal{O}\left(\alpha_{s}\right)$ QCD corrections, $\delta_{\mathrm{w}}$ contains the purely weak correction at one loop, $\delta_{x_{t}}$ is the leading $\mathcal{O}\left(x_{t}\right)$ term of the latter, the factor $\delta_{t}$ supplies the leading topquark-induced corrections at one loop and beyond, and $\delta_{\text {res }}$ comprises the residual higher-order corrections, which are beyond the scope of our present analysis.

It is useful to distinguish between the class of contributions devoid of the $H f \bar{f}$ Yukawa coupling and the complementary class. The former survives in the massless limit $m \rightarrow 0$, and the parts of $\delta_{0}$ and $\delta_{1}$ that belong to it are related via the optical theorem to the absorptive part of the $Z$-boson vacuum polarization tensor induced by the fermion $f$,

$$
\Pi^{\mu \nu}(q)=g^{\mu \nu} \Pi_{T}(s)+q^{\mu} q^{\nu} \Pi_{L}(s),
$$

where

$$
\Pi_{i}(s)=\frac{G_{F} z}{2^{3 / 2}}\left[V^{2} \Pi_{i}^{V}(s)+A^{2} \Pi_{i}^{A}(s)\right] \quad(i=T, L) .
$$

While, for $m=0$, only the transversal part $\Pi_{T}(s)$ matters, the longitudinal part $\Pi_{L}(s)$, too, is relevant for $m>0$. Due to vector current conservation, we have

$$
\Pi_{T}^{V}(s)+s \Pi_{L}^{V}(s)=0,
$$

so that we have to consider only three different coefficient functions. Through the two-loop order, we have [56-58]

$$
\begin{aligned}
& \operatorname{Im} \Pi_{T}^{V}(s)=\frac{s}{12 \pi} N\left[v_{0}(r)+\frac{3}{4}\left(\frac{\alpha}{\pi} Q^{2}+\frac{\alpha_{s}}{\pi} C_{F}\right) v_{1}(r)\right] \\
& \operatorname{Im} \Pi_{T}^{A}(s)=\frac{s}{12 \pi} N\left[a_{0}(r)+\frac{3}{4}\left(\frac{\alpha}{\pi} Q^{2}+\frac{\alpha_{s}}{\pi} C_{F}\right) a_{1}(r)\right] \\
& \operatorname{Im} \Pi_{L}^{A}(s)=\operatorname{Im}_{L}^{V}(s)-\frac{1}{12 \pi} N\left[l_{0}(r)+\frac{3}{4}\left(\frac{\alpha}{\pi} Q^{2}+\frac{\alpha_{s}}{\pi} C_{F}\right) l_{1}(r)\right],
\end{aligned}
$$

where

$$
v_{0}(r)=\rho\left(1+\frac{1}{2 r}\right), \quad a_{0}(r)=\rho^{3}, \quad l_{0}(r)=0,
$$

$r v_{1}(r)$ and $r a_{1}(r)$ are given by Eqs. (5) and (6) in Ref. [57], respectively, $-l_{1}(r) /(4 \pi)$ is given by the second term on the right-hand side of Eq. (13) in Ref. [58], $r=s /\left(4 m^{2}\right)$, and $\rho=\sqrt{1-1 / r}$. In Eq. (7), the normalizations are arranged so that $v_{i}(\infty)=a_{i}(\infty)=1(i=0,1)$, while we have $l_{1}(r)=1 / r^{2}+\mathcal{O}\left(1 / r^{3}\right)$ for $r \gg 1$.

The correction terms in Eq. (2) may now be presented in a compact form. Specifically, we have

$$
\begin{aligned}
\delta_{i}= & \frac{1}{V^{2}+A^{2}}\left\{V^{2} v_{i}(r)+A^{2} a_{i}(r)+\frac{(s-z)^{2}}{C_{1}}\right. \\
& \left.\times\left[-\frac{\lambda^{3 / 2}}{3 z^{3}} A^{2}\left(a_{i}(r)-v_{i}(r)-l_{i}(r)\right)+y_{i}\right]\right\},
\end{aligned}
$$

where $y_{0}$ and $y_{1}$ represent, respectively, the tree-level and one-loop corrections involving one or two powers of the $H f \bar{f}$ Yukawa coupling. For $m=0$, we have $\delta_{i}=1$, so that $\delta_{i}-1$ measures the relative finite- $m$ correction at tree level $(i=0)$ or at one loop in QED and QCD $(i=1)$. For $y_{0}$, we find

$$
\begin{aligned}
y_{0}= & \frac{2 m^{2}}{z^{2}(z-s)}\left\{\rho \sqrt{\lambda}\left[-\left(V^{2}\left(z+2 m^{2}\right)+A^{2}\left(z-4 m^{2}\right)\right) \frac{s(z-s)\left(h-4 m^{2}\right)}{h z s+m^{2} \lambda}-4 V^{2} z+A^{2}\left(6 z+s-\frac{s}{z}(2 h-s)\right)\right]\right. \\
& +V^{2} L\left[3 z^{2}-z(7 h-6 s)-2 h^{2}-h s-s^{2}+4 m^{2}(5 h+2 s)-16 m^{4}+2 h \frac{h^{2}-2 m^{2}(5 h-2 s)+8 m^{4}}{h+z-s}\right] \\
& +A^{2} L\left[3 z^{2}+z(h+6 s)+(h-s)(2 h+s)-2 m^{2}\left(13 z-h+6 s+\frac{(h-s)(2 h-s)}{z}\right)\right. \\
& \left.\left.+32 m^{4}-2 h \frac{h^{2}-4 m^{2}(h+s)+16 m^{4}}{h+z-s}\right]\right\},
\end{aligned}
$$

where

$$
L=\ln \frac{h+z-s-\rho \sqrt{\lambda}}{h+z-s+\rho \sqrt{\lambda}} .
$$

As we shall see in Sec. III, the contribution to $\delta_{0}$ proportional to $y_{0}$ is exceedingly small. Our result for $y_{1}$ is too lengthy to be presented here. 
Furthermore, we have

$$
\delta_{\mathrm{w}}=2 \operatorname{Re} \Delta_{\text {weak }}+\delta_{m},
$$

where the $m=0$ part $\Delta_{\text {weak }}$ is listed in analytic form in Ref. [50], and $\delta_{m}$ comprises the finite- $m$ correction. We include $\delta_{m}$ in our numerical analysis, although it turns out to be small against $\Delta_{\text {weak }}$, but we refrain from presenting here our analytic expression for it because it is too lengthy.

Finally, using the improved Born approximation (IBA) [59], we obtain

$$
\begin{aligned}
\delta_{t}= & \frac{\left(1+\delta_{Z Z H}\right)^{2}}{1-\Delta \rho} \frac{\left(V-4 c_{w}^{2} Q \Delta \rho\right)^{2}+A^{2}}{V^{2}+A^{2}} \\
= & 1+2 \delta_{Z Z H}+(1-8 X) \Delta \rho+\delta_{Z Z H}^{2}+2(1 \\
& -8 X) \delta_{Z Z H} \Delta \rho+(1-8 X+16 Y)(\Delta \rho)^{2}+\mathcal{O}\left(x_{t}^{3}\right),
\end{aligned}
$$

where $\left(1+\delta_{Z Z H}\right)$ is the correction to the heavy-topquark effective Lagrangian of the $Z Z H$ interaction, $\Delta \rho=1-1 / \rho$ measures the deviation of the electroweak $\rho$ parameter from unity, $X=c_{w}^{2} Q V /\left(V^{2}+A^{2}\right)$, and $Y=c_{w}^{4} Q^{2} /\left(V^{2}+A^{2}\right)$. Including the corrections of $\mathcal{O}\left(x_{t}\right)$ $[25,44,45,60,61], \mathcal{O}\left(x_{t}^{2}\right)[21,62], \mathcal{O}\left(x_{t} \alpha_{s}\right)$ [48,63], and $\mathcal{O}\left(x_{t} \alpha_{s}^{2}\right)[38,64]$, we have

$$
\begin{aligned}
\delta_{\text {ZZH }}= & x_{t}\left\{-\frac{5}{2}-\left[\frac{177}{8}+18 \zeta(2)\right] x_{t}\right. \\
& \left.+[15-2 \zeta(2)] a_{s}+17.117 a_{s}^{2}\right\}, \\
\Delta \rho= & x_{t}\left\{3+3[19-12 \zeta(2)] x_{t}\right. \\
& \left.-2[1+2 \zeta(2)] a_{s}-43.782 a_{s}^{2}\right\},
\end{aligned}
$$

where $\zeta(2)=\pi^{2} / 6$ and $a_{s}=\alpha_{s}^{(6)}\left(m_{t}\right) / \pi$. Analytic expressions for the $\mathcal{O}\left(x_{t} \alpha_{s}^{2}\right)$ terms in Eq. (14) may be found in Refs. [38,64]. To avoid double counting, the $\mathcal{O}\left(x_{t}\right)$ term of Eq. (13) [50,53],

$$
\delta_{x_{t}}=-2 x_{t}(1+12 X),
$$

is subtracted in Eq. (2).

A more conservative form of Eq. (2) is obtained by discarding the quantities $\delta_{x_{t}}$ and $\delta_{t}$, and in turn introducing within the square brackets the term $\delta_{\text {ho }}$ that contains the leading top-quark-induced corrections beyond one loop. In the leptonic case $f=l$, which is of special interest here, we have

$$
\begin{aligned}
\delta_{\mathrm{ho}}= & \delta_{t}-1-\delta_{x_{t}} \\
= & \{13-72 \zeta(2)+24[-17+12 \zeta(2)] X \\
& +144 Y\} x_{t}^{2}+4\{7-2 \zeta(2)+4[1+2 \zeta(2)] X\} x_{t} a_{s} \\
& +(-9.548+350.257 X) x_{t} a_{s}^{2} .
\end{aligned}
$$

Finally, $\Gamma_{H \rightarrow Z f \bar{f}}$ is obtained by integrating Eq. (2) over the interval $4 m^{2}<s<\left(m_{H}-m_{Z}\right)^{2}$. The tree-level result for $m=0$ reads [55]

$$
\Gamma_{H \rightarrow Z f \bar{f}}^{0}=\frac{G_{F}^{2} z^{4}}{128 \pi^{3} h^{3 / 2}} N\left(V^{2}+A^{2}\right)\left[-F\left(\frac{h}{z}\right)\right],
$$

where $F(x)$ is given in Eq. (3) of Ref. [53]. ${ }^{1}$ For the reader's convenience, we reproduce this formula here:

$$
\begin{aligned}
F(x)= & \frac{1-x}{x}\left(-\frac{47}{2}+\frac{13}{2} x-x^{2}\right)+\left(-2+3 x-\frac{x^{2}}{2}\right) \ln x \\
& +\left(10-4 x+\frac{x^{2}}{2}\right) \sqrt{\frac{x}{4-x}}\left(\pi-6 \arcsin \frac{\sqrt{x}}{2}\right) .
\end{aligned}
$$

The origin of the minus sign on the right-hand side of Eq. (17) is explained in Eq. (1).

\section{B. $\boldsymbol{H} \rightarrow \boldsymbol{W}^{+} \boldsymbol{f} \bar{f}^{\prime}$ decay}

We now consider the decay process $H \rightarrow W^{+} f \bar{f}^{\prime}$, where $f$ is a generic fermion with weak isospin $I=-1 / 2$ and $f^{\prime}$ is an appropriate fermion with $I=1 / 2$. By chargeconjugation invariance, the process $H \rightarrow W^{-} f^{\prime} \bar{f}$ has the same partial decay width.

Some of the expressions for $H \rightarrow Z f \bar{f}$ in Sec. II A carry over to $H \rightarrow W^{+} f \bar{f}^{\prime}$. Specifically, the counterparts of Eqs. (1), (3)-(5), and (17), are obtained by substituting $z \rightarrow w$ and $V, A \rightarrow \sqrt{2}$, and including the overall factor $\left|V_{f^{\prime} f}\right|^{2}$, where $V_{f^{\prime} f}$ is the Cabibbo-Kobayashi-Maskawa quark mixing matrix, if $f$ and $f^{\prime}$ are quarks. However, structural differences occur because the intermediate boson is now electrically charged. In particular, the separation of QED and weak corrections is no longer meaningful at one loop because the photonic loop diagrams, including the appropriate counterterm diagrams, no longer form a gauge-independent and ultraviolet-finite subset $[15,43]$. Furthermore, in the case when $f$ and $f^{\prime}$ are quarks, the one-loop QCD correction is no longer proportional to the QED correction. As a consequence, the distribution of the partial decay width $\Gamma_{H \rightarrow W^{+} f \bar{f}^{\prime}}$ in the $f \bar{f}^{\prime}$ invariant mass square $s=q^{2}$ now takes the form

$$
\begin{aligned}
\frac{d \Gamma_{H \rightarrow W^{+} f \bar{f}^{\prime}}}{d s}= & \frac{d \Gamma_{H \rightarrow W^{+} f \bar{f}^{\prime}}^{0}\left(\delta_{0}+\frac{3}{4} \frac{\alpha_{s}}{\pi} C_{F} \delta_{1}\right.}{d s} \\
& \left.+\delta_{\text {ew }}-\delta_{x_{t}}+\delta_{\text {res }}\right) \delta_{t} .
\end{aligned}
$$

In view of $m_{\nu} \ll m_{\ell}$ and $m_{s} \ll m_{c}$, we may safely neglect the mass of the lighter one of the two fermions $f$ and $f^{\prime}$ and call the mass of the heavier one $m$. Due to the $\gamma_{5}$ reflection property $\Pi_{i}^{V}\left(s, m_{1}, m_{2}\right)=\Pi_{i}^{A}\left(s, m_{1},-m_{2}\right)$ $(i=T, L)$, we then have [56-58]

$$
\Pi_{i}^{V}(s)=\Pi_{i}^{A}(s) .
$$

Through the two-loop order, we have [56-58]

\footnotetext{
${ }^{1}$ In the journal publication [53], this equation contains a misprint, which is absent in the preprint: $\sqrt{\frac{1}{4} x-x}$ should replaced by $\sqrt{\frac{x}{4-x}}$.
} 


$$
\begin{aligned}
& \operatorname{Im} \Pi_{T}^{V}(s)=\frac{s}{12 \pi} N\left[f_{0}(x)+\frac{3}{4} \frac{\alpha_{s}}{\pi} C_{F} f_{1}(x)\right], \\
& \operatorname{Im} \Pi_{L}^{V}(s)=-\frac{1}{12 \pi} N\left[g_{0}(x)+\frac{3}{4} \frac{\alpha_{s}}{\pi} C_{F} g_{1}(x)\right],
\end{aligned}
$$

where

$$
\begin{aligned}
& f_{0}(x)=\left(1+\frac{1}{2 x}\right)\left(1-\frac{1}{x}\right)^{2}, \\
& g_{0}(x)=\left(1+\frac{2}{x}\right)\left(1-\frac{1}{x}\right)^{2},
\end{aligned}
$$

$x f_{1}(x) / 4$ is given by Eq. (7) in Ref. [57], $-g_{1}(x) / 4$ is given by Eq. (14) in Ref. [58], and $x=s / m^{2}$. In Eq. (7), the normalizations are arranged so that $f_{i}(\infty)=g_{i}(\infty)=1$ $(i=0,1)$.

Then, we have

$$
\delta_{i}=f_{i}(x)+\frac{(s-w)^{2}}{C_{1}}\left\{-\frac{\lambda^{3 / 2}}{3 w^{3}}\left[f_{i}(x)-g_{i}(x)\right]+y_{i}\right\},
$$

where $C_{1}$ and $\lambda$ are defined as below Eq. (3), but with $z$ replaced by $w$. For $y_{0}$, we find

$$
\begin{aligned}
y_{0}= & \frac{m^{2}}{2 w^{3} s^{2}(w-s)}\left\{\sqrt { \lambda } \left[s^{3}(3 w+s)-2 h\left(s-m^{2}\right)\left(s^{2}+2 m^{2}(w-s)\right)+2 m^{2} w(w+s)\left(s-2 m^{2}\right)\right.\right. \\
& \left.+\frac{m^{2} s^{2}(w-s)\left(2 w+m^{2}\right)\left(w+s-2 m^{2}\right)^{2}}{h\left(w-m^{2}\right)\left(s-m^{2}\right)+m^{2}(w-s)^{2}}\right]+s^{2} L[2 w(3 w(-h+w+2 s)-s(h+s)) \\
& \left.\left.+m^{2}(w(9 h-5 w-2 s)+s(3 h-s))-4 m^{4}(h+w+s)\right]\right\}
\end{aligned}
$$

where

$$
L=\ln \frac{\left(s+m^{2}\right)(w-s)+\left(s-m^{2}\right)(h-\sqrt{\lambda})}{\left(s+m^{2}\right)(w-s)+\left(s-m^{2}\right)(h+\sqrt{\lambda})} .
$$

As will be shown in Sec. III, the contribution proportional to $y_{0}$ is very small compared to $\delta_{0}$. For lack of space, we do not present $y_{1}$ and $\delta_{\text {ew }}$ in analytic form. Furthermore, we have

$$
\delta_{t}=\left(1+\delta_{W W H}\right)^{2} .
$$

Including the corrections of $\mathcal{O}\left(x_{t}\right)[25,43,45,60], \mathcal{O}\left(x_{t}^{2}\right)$ [21], $\mathcal{O}\left(x_{t} \alpha_{s}\right)$ [47], and $\mathcal{O}\left(x_{t} \alpha_{s}^{2}\right)$ [38], we have

$$
\begin{aligned}
\delta_{W W H}= & x_{t}\left\{-\frac{5}{2}+\left[\frac{39}{8}-18 \zeta(2)\right] x_{t}\right. \\
& \left.+[9-2 \zeta(2)] a_{s}+27.041 a_{s}^{2}\right\} .
\end{aligned}
$$

An analytic expression for the $\mathcal{O}\left(x_{t} \alpha_{s}^{2}\right)$ term may be found in Ref. [38]. The $\mathcal{O}\left(x_{t}\right)$ term of Eq. (26) is

$$
\delta_{x_{t}}=-5 x_{t} .
$$

Again, we may trade the quantities $\delta_{x_{t}}$ and $\delta_{t}$ in Eq. (19) against the term $\delta_{\text {ho }}$, carrying the leading top-quarkinduced corrections beyond one loop, to be inserted within the parentheses. In the leptonic case, we have

$$
\begin{aligned}
\delta_{\mathrm{ho}} & =\delta_{t}-1-\delta_{x_{t}} \\
& =4[4-9 \zeta(2)] x_{t}^{2}+2[9-2 \zeta(2)] x_{t} a_{s}+54.082 x_{t} a_{s}^{2} .
\end{aligned}
$$

Finally, $\Gamma_{H \rightarrow W^{+} f \bar{f}^{\prime}}$ is obtained by integrating Eq. (19) over the interval $m^{2}<s<\left(m_{H}-m_{W}\right)^{2}$.

\section{NUMERICAL RESULTS}

We are now in a position to explore the phenomenological consequences of our results by performing a numerical analysis. We adopt all the input parameter values from Ref. [65]. Specifically, we use $m_{W}=80.385 \mathrm{GeV}$, $m_{Z}=91.1876 \mathrm{GeV}, m_{b}=4.78 \mathrm{GeV}, m_{t}=173.5 \mathrm{GeV}$, and $\alpha_{s}^{(6)}\left(m_{t}\right)=0.1080$, which follows from $\alpha_{s}^{(5)}\left(m_{Z}\right)=$ 0.1184 via four-loop evolution and three-loop matching [66]. In our renormalization scheme, $\sin ^{2} \theta_{\mathrm{w}}=$ $1-m_{W}^{2} / m_{Z}^{2}=0.222897$ and $\alpha=\sqrt{2} G_{F} \sin ^{2} \theta_{\mathrm{w}} m_{W}^{2} / \pi=$ $1 / 132.233$ are derived parameters.

We first consider the $H \rightarrow Z \ell^{+} \ell^{-}$decays. In Fig. 1, we present the tree-level finite- $m$ (dotted lines) and radiative (solid lines) corrections to (a) $d \Gamma\left(H \rightarrow Z \tau^{+} \tau^{-}\right) / d s$ for $m_{H}=125 \mathrm{GeV}$ as functions of $\sqrt{s}$, and to (b) $\Gamma\left(H \rightarrow Z \tau^{+} \tau^{-}\right)$and (c) $\Gamma\left(H \rightarrow Z \mu^{+} \mu^{-}\right)$as functions of $m_{H}$. The radiative corrections are decomposed into the $\mathcal{O}(\alpha)$ QED (coarsely dotted lines), $\mathcal{O}(\alpha)$ weak (dashed lines), and dominant higher-order (dot-dashed lines) corrections of $\mathcal{O}\left(x_{t}^{2}\right), \mathcal{O}\left(x_{t} \alpha_{s}\right)$, and $\mathcal{O}\left(x_{t} \alpha_{s}^{2}\right)$. For comparison, the $\mathcal{O}(\alpha)$ corrections predicted by the IBA (dot-dot-dashed lines) are also shown. Looking at Fig. 1(a), we observe that the QED correction $3 \alpha \delta_{1} /(4 \pi)$ exhibits an enhancement towards low values of $\sqrt{s}$, which is of Coulomb origin, while it gets close to its asymptotic value $3 \alpha /(4 \pi)$ as $\sqrt{s}$ approaches its kinematic upper bound. The purely weak correction $\delta_{\mathrm{w}}$ monotonically increases with $\sqrt{s}$, ranging from slightly negative values at the $\tau^{+} \tau^{-}$pair production threshold to about $2.0 \%$ at the upper endpoint. The oneloop electroweak correction is inadequately described by the IBA term $\delta_{x_{t}}$. The dominant higher-order correction $\delta_{\text {ho }}$ amounts to about $0.2 \%$ altogether and incidentally almost coincides with the QED correction. The finite- $m$ 


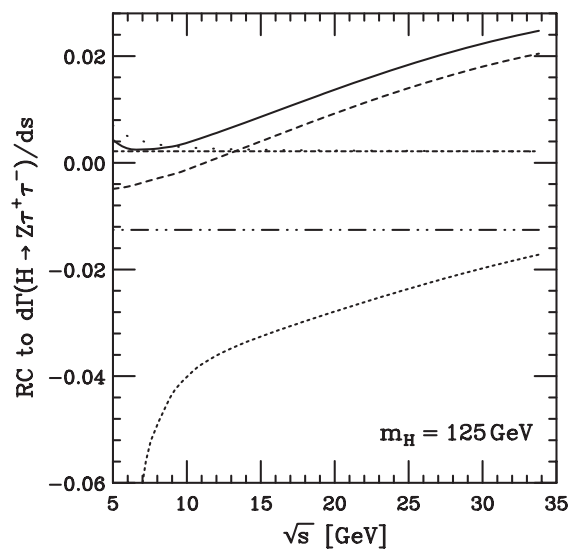

(a)

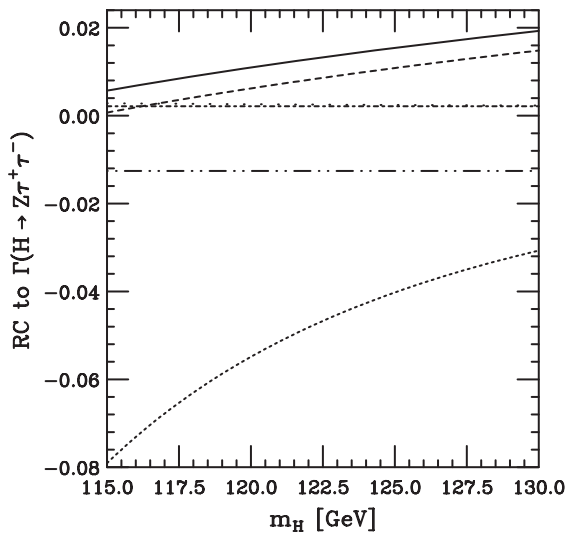

(b)

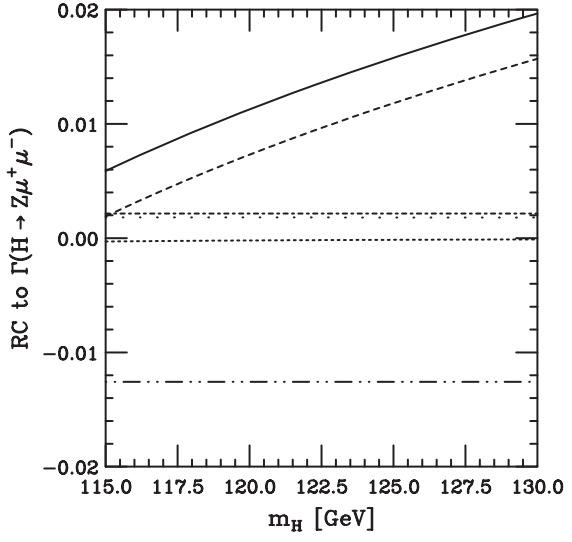

(c)

FIG. 1. Tree-level finite- $m$ (dotted lines) and radiative (solid lines) corrections to (a) $d \Gamma\left(H \rightarrow Z \tau^{+} \tau^{-}\right) / d s$ for $m_{H}=125 \mathrm{GeV}$ as functions of the $\tau^{+} \tau^{-}$invariant mass $\sqrt{s}$, and to (b) $\Gamma\left(H \rightarrow Z \tau^{+} \tau^{-}\right)$and (c) $\Gamma\left(H \rightarrow Z \mu^{+} \mu^{-}\right)$as functions of $m_{H}$. The radiative corrections include the $\mathcal{O}(\alpha)$ QED (coarsely dotted lines), $\mathcal{O}(\alpha)$ weak (dashed lines), and dominant higher-order (dot-dashed lines) corrections of $\mathcal{O}\left(x_{t}^{2}\right), \mathcal{O}\left(x_{t} \alpha_{s}\right)$, and $\mathcal{O}\left(x_{t} \alpha_{s}^{2}\right)$. For comparison, the $\mathcal{O}(\alpha)$ corrections predicted by the IBA (dot-dot-dashed lines) are also shown.

correction $\delta_{0}-1$, of course, quenches $d \Gamma\left(H \rightarrow Z \tau^{+} \tau^{-}\right) /$ $d s$ at threshold, but it is still as large as $-1.7 \%$ at the upper endpoint, largely compensating the combined radiative correction. As anticipated in Sec. II A, the relative contribution of $y_{0}$ to $\delta_{0}$, proportional to the $H \tau^{+} \tau^{-}$Yukawa coupling, is exceedingly small in magnitude, below $0.09 \%$, over the full $\sqrt{s}$ range. The finite- $m$ corrections to $d \Gamma(H \rightarrow$ $\left.Z \mu^{+} \mu^{-}\right) / d s$ and $d \Gamma\left(H \rightarrow Z e^{+} e^{-}\right) / d s$ are negligible compared to the expected size of the presently unknown subleading higher-order corrections $\delta_{\text {res }}$, and the radiative corrections to both observables are practically indistinguishable thanks to the almost perfect lepton universality. The latter are also very similar to the radiative corrections to $d \Gamma\left(H \rightarrow Z \tau^{+} \tau^{-}\right) / d s$, and we refrain from presenting the counterparts of Fig. 1(a) for $d \Gamma\left(H \rightarrow Z \mu^{+} \mu^{-}\right) / d s$ and $d \Gamma\left(H \rightarrow Z e^{+} e^{-}\right) / d s$.

Looking at Fig. 1(b), we observe that the finite- $m$ correction to $\Gamma\left(H \rightarrow Z \tau^{+} \tau^{-}\right)$ranges from $-7.9 \%$ to $-3.1 \%$ in the considered mass window $115 \mathrm{GeV}<m_{H}<$ $130 \mathrm{GeV}$ and more than compensates the overall radiative correction, which ranges there between $0.6 \%$ and $1.9 \%$. From Fig. 1(c), we read off that the finite- $m$ correction to $\Gamma\left(H \rightarrow Z \mu^{+} \mu^{-}\right)$is below $0.03 \%$ in magnitude.

In Fig. 2(a), we present our best predictions for $d \Gamma\left(H \rightarrow Z \tau^{+} \tau^{-}\right) / d s$ (solid line) and $d \Gamma\left(H \rightarrow Z \mu^{+} \mu^{-}\right) /$ $d s$ (dashed line) for $m_{H}=125 \mathrm{GeV}$ as functions of $\sqrt{s}$

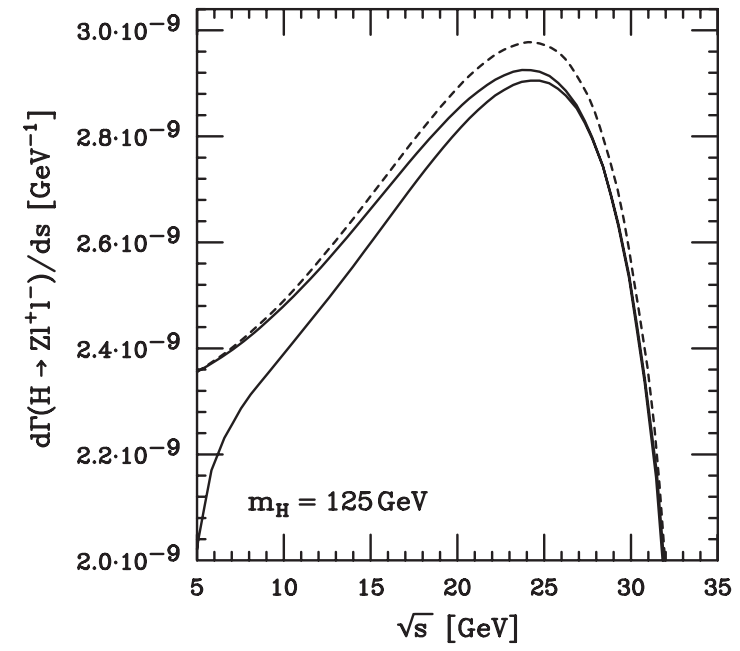

(a)

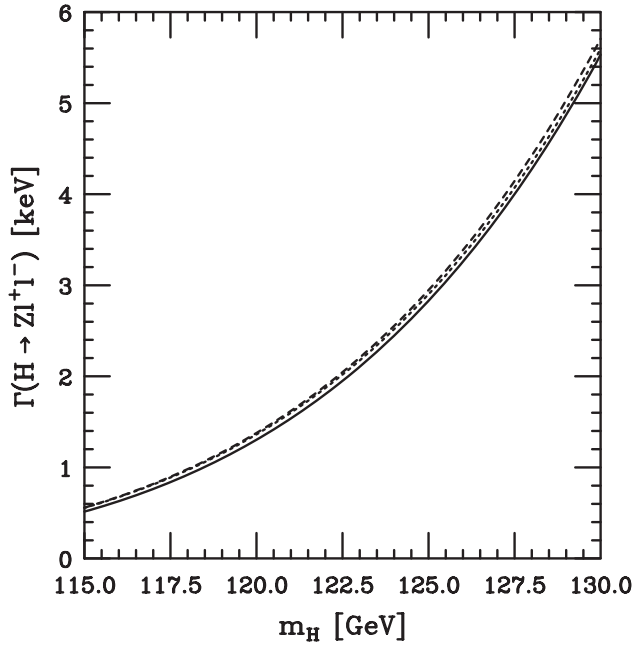

(b)

FIG. 2. (a) $d \Gamma\left(H \rightarrow Z \ell^{+} \ell^{-}\right) / d s$ for $m_{H}=125 \mathrm{GeV}$ as a function of the $\ell^{+} \ell^{-}$invariant mass $\sqrt{s}$, and (b) $\Gamma\left(H \rightarrow Z \ell^{+} \ell^{-}\right)$as a function of $m_{H}$ at the tree level for $m=0$ (dotted lines) and including both finite- $m$ and radiative corrections for $\ell=\mu$ (dashed lines) and $\ell=\tau$ (solid lines). 


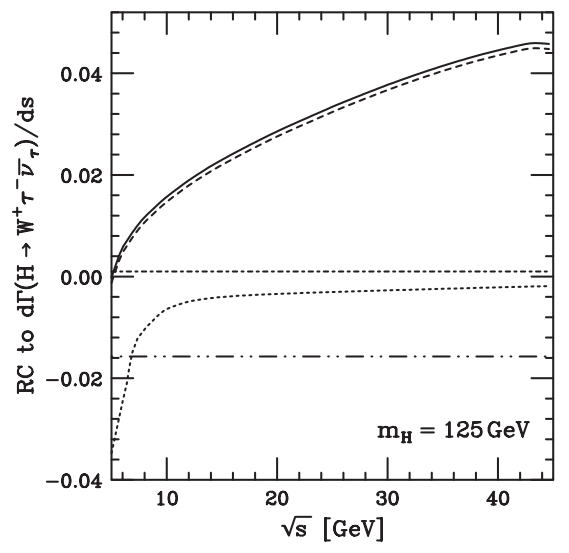

(a)

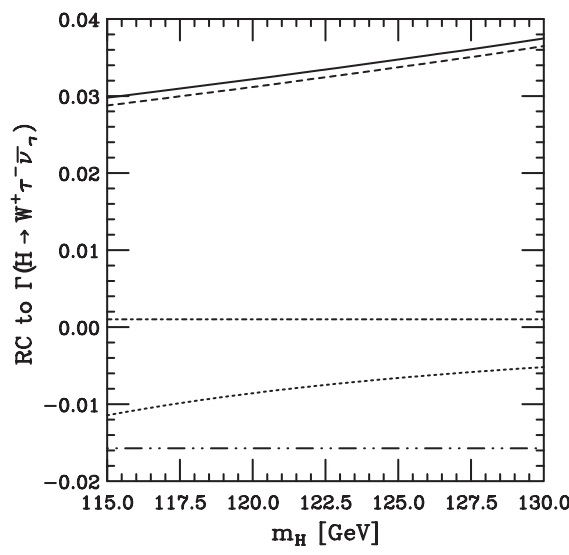

(b)

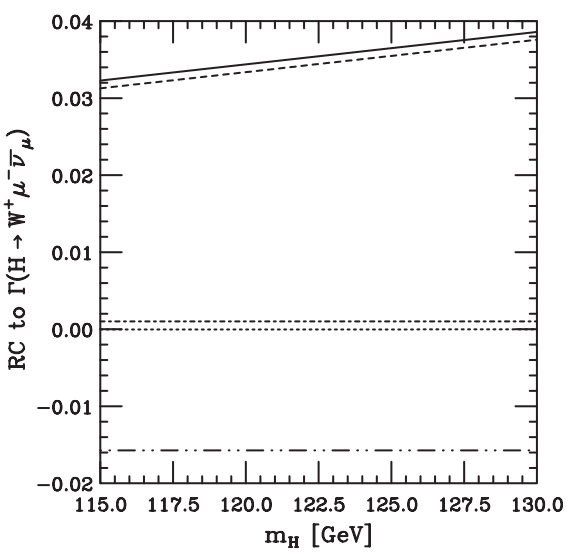

(c)

FIG. 3. Tree-level finite- $m$ (dotted lines) and radiative (solid lines) corrections to (a) $d \Gamma\left(H \rightarrow W^{+} \tau^{-} \bar{\nu}_{\tau}\right) / d s$ for $m_{H}=125 \mathrm{GeV}$ as functions of the $\tau^{-} \bar{\nu}_{\tau}$ invariant mass $\sqrt{s}$, and to (b) $\Gamma\left(H \rightarrow W^{+} \tau^{-} \bar{\nu}_{\tau}\right)$ and (c) $\Gamma\left(H \rightarrow W^{+} \mu^{-} \bar{\nu}_{\mu}\right)$ as functions of $m_{H}$. The radiative corrections include the $\mathcal{O}(\alpha)$ electroweak (dashed lines) and dominant higher-order (dot-dashed lines) corrections of $\mathcal{O}\left(x_{t}^{2}\right), \mathcal{O}\left(x_{t} \alpha_{s}\right)$, and $\mathcal{O}\left(x_{t} \alpha_{s}^{2}\right)$. For comparison, the $\mathcal{O}(\alpha)$ corrections predicted by the IBA (dot-dot-dashed lines) are also shown.

including both finite- $m$ and radiative corrections. For comparison, the tree-level result for $m=0$ (dotted line) is also shown. The relation of the solid line shape to the dotted one may be easily understood from Fig. 1(a). The essential feature of the dashed line shape in comparison to the solid one is the insignificance of the finite- $m$ correction for $\ell=\mu$ already mentioned above.

In Fig. 2(b), our best predictions for $\Gamma\left(H \rightarrow Z \tau^{+} \tau^{-}\right)$ (solid line) and $\Gamma\left(H \rightarrow Z \mu^{+} \mu^{-}\right)$(dashed line) as functions of $m_{H}$ are compared with the tree-level result for $m=0$ (dotted line). The relative shifts of the solid and dashed line shapes with respect to the dotted one immediately follow from Figs. 1(b) and 1(c), respectively.
We now turn to the $H \rightarrow W^{+} \ell^{-} \bar{\nu}_{\ell}$ decays. Figure 3 shows the tree-level finite- $m$ (dotted lines) and radiative (solid lines) corrections to (a) $d \Gamma\left(H \rightarrow W^{+} \tau^{-} \bar{\nu}_{\tau}\right) / d s$ for $m_{H}=125 \mathrm{GeV}$ as functions of $\sqrt{s}$, and to (b) $\Gamma\left(H \rightarrow W^{+} \tau^{-} \bar{\nu}_{\tau}\right)$ and (c) $\Gamma\left(H \rightarrow W^{+} \mu^{-} \bar{\nu}_{\mu}\right)$ as functions of $m_{H}$. The radiative corrections are built up by the $\mathcal{O}(\alpha)$ electroweak (dashed lines) and dominant higherorder (dot-dashed lines) corrections of $\mathcal{O}\left(x_{t}^{2}\right), \mathcal{O}\left(x_{t} \alpha_{s}\right)$, and $\mathcal{O}\left(x_{t} \alpha_{s}^{2}\right)$. For comparison, the $\mathcal{O}(\alpha)$ corrections predicted by the IBA (dot-dot-dashed lines) are also presented. Looking at Fig. 3(a), we observe that the electroweak correction $\delta_{\text {ew }}$ monotonically increases with $\sqrt{s}$, ranging from slightly negative values at the $\tau^{-} \bar{\nu}_{\tau}$ pair production

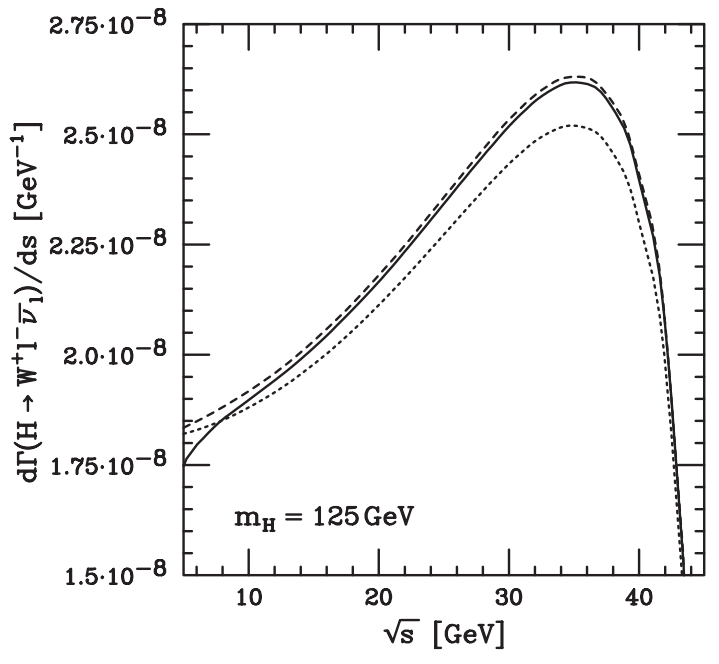

(a)

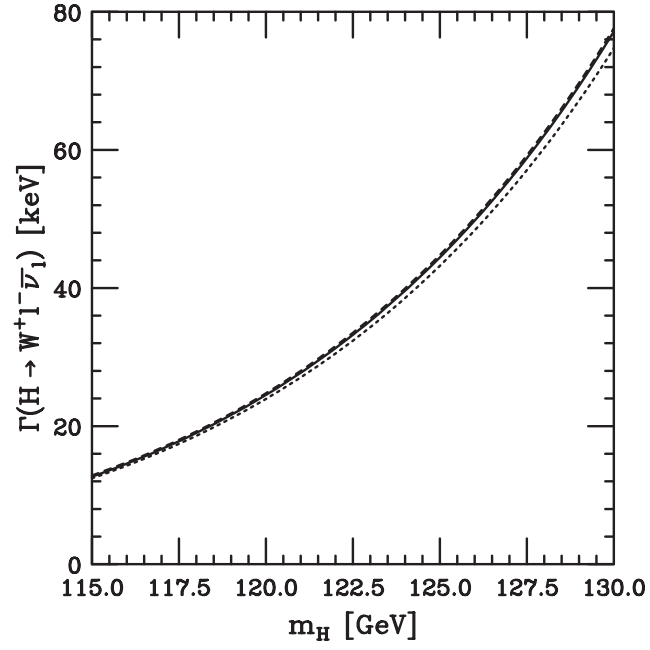

(b)

FIG. 4. (a) $d \Gamma\left(H \rightarrow W^{+} \ell^{-} \bar{\nu}_{\ell}\right) / d s$ for $m_{H}=125 \mathrm{GeV}$ as a function of the $\ell^{-} \bar{\nu}_{\ell}$ invariant mass $\sqrt{s}$, and (b) $\Gamma\left(H \rightarrow W^{+} \ell^{-} \bar{\nu}_{\ell}\right)$ as a function of $m_{H}$ at the tree level for $m=0$ (dotted lines) and including both finite- $m$ and radiative corrections for $\ell=\mu$ (dashed lines) and $\ell=\tau$ (solid lines). 
threshold to about $4.5 \%$ at the upper endpoint. The oneloop electroweak correction is again inadequately described by the IBA term $\delta_{x_{t}}$. The dominant higher-order correction $\delta_{\text {ho }}$ amounts to about $0.1 \%$ altogether.

Comparing Figs. 3(a)-3(c) with Figs. 1(a)-1(c), we observe that the finite- $m$ corrections for the $H \rightarrow W^{+} \ell^{-} \bar{\nu}_{\ell}$ decays are significantly smaller than for the respective $H \rightarrow Z \ell^{+} \ell^{-}$decays. This is mainly due to the fact that the shrinkage of the available phase space caused by switching on the finite- $m$ corrections is lesser for the $H \rightarrow W^{+} \ell^{-} \bar{\nu}_{\ell}$ decays because the lepton pair production threshold is twice as low, at $\sqrt{s}=m$, and the upper endpoint is located higher, by $m_{Z}-m_{W} \approx 11 \mathrm{GeV}$, also leading to correspondingly higher maxima of the $\sqrt{s}$ distributions. In fact, the finite- $m$ correction $\delta_{0}-1$ to $d \Gamma(H \rightarrow$ $\left.W^{+} \tau^{-} \bar{\nu}_{\tau}\right) / d s$ in Fig. 3(a) rapidly relaxes from its threshold value of $-100 \%$ to moderate values, passing $-1 \%$ at $\sqrt{s} \approx$ $8 \mathrm{GeV}$ and reaching $-0.2 \%$ at the upper endpoint. As anticipated in Sec. II B, the relative contribution of $y_{0}$ to $\delta_{0}$, proportional to the $H \tau^{+} \tau^{-}$Yukawa coupling, is very small, below $0.17 \%$, over the full $\sqrt{s}$ range.

From Fig. 3(b), we learn that the finite- $m$ correction to $\Gamma\left(H \rightarrow W^{+} \tau^{-} \bar{\nu}_{\tau}\right)$ ranges from $-1.1 \%$ to $-0.5 \%$ in the considered mass window $115 \mathrm{GeV}<m_{H}<130 \mathrm{GeV}$ and reduces the radiative corrections by between $38 \%$ and 14\%. On the other hand, Fig. 3(c) tells us that the finite- $m$ correction to $\Gamma\left(H \rightarrow W^{+} \mu^{-} \bar{\nu}_{\mu}\right)$ is absolutely negligible, being below $0.004 \%$ in magnitude.

In Fig. 4(a), our final predictions for $d \Gamma\left(H \rightarrow W^{+} \tau^{-} \bar{\nu}_{\tau}\right) /$ $d s$ (solid line) and $d \Gamma\left(H \rightarrow W^{+} \mu^{-} \bar{\nu}_{\mu}\right) / d s$ (dashed line) for $m_{H}=125 \mathrm{GeV}$ are shown as functions of $\sqrt{s}$ and compared with the tree-level result for $m=0$ (dotted line), so as to expose the interplay of the finite- $m$ and radiative corrections. In Fig. 4(b), the same is done for $\Gamma\left(H \rightarrow W^{+} \tau^{-} \bar{\nu}_{\tau}\right)$ and $\Gamma\left(H \rightarrow W^{+} \mu^{-} \bar{\nu}_{\mu}\right)$ as functions of $m_{H}$.

\section{CONCLUSIONS}

We presented a precision study of the partial widths of the $H \rightarrow Z \ell^{+} \ell^{-}$and $H \rightarrow W^{+} \ell^{-} \bar{\nu}_{\ell}$ decays, including the full one-loop electroweak corrections and the dominant contributions at two and three loops, of $\mathcal{O}\left(x_{t}^{2}\right), \mathcal{O}\left(x_{t} \alpha_{s}\right)$, and $\mathcal{O}\left(x_{t} \alpha_{s}^{2}\right)$. We also included finite- $m$ corrections, which turned out to be indispensable for $\ell=\tau$, but negligible for $\ell=\mu, e$. Working in the on-mass-shell renormalization scheme with $G_{F}$ replacing $\alpha$ as a basic parameter, we ensured that the radiative corrections remained moderate in size, being devoid of large logarithms involving small masses of charged fermions. As for the integrated partial decay widths, we found the net corrections relative to the tree-level results for $m=0$ [55] at $m_{H}=125 \mathrm{GeV}$ to be $+1.6 \%$ for $H \rightarrow Z e^{+} e^{-}$and $H \rightarrow Z \mu^{+} \mu^{-},-2.5 \%$ for $H \rightarrow Z \tau^{+} \tau^{-}, \quad+3.6 \%$ for $H \rightarrow W^{+} e^{-} \bar{\nu}_{e}$ and $H \rightarrow W^{+} \mu^{-} \bar{\nu}_{\mu}$, and $+2.8 \%$ for $H \rightarrow W^{+} \tau^{-} \bar{\nu}_{\tau}$.

\section{ACKNOWLEDGMENTS}

This work was supported in part by the German Federal Ministry for Education and Research BMBF through Grant No. 05H12GUE and by the Helmholtz Association HGF through Grant No. Ha 101.
[1] G. Aad et al. (ATLAS Collaboration), Phys. Lett. B 710, 49 (2012); P. Mal (on behalf of the ATLAS Collaboration), arXiv:1206.1174.

[2] S. Chatrchyan et al. (CMS Collaboration), Phys. Lett. B 710, 26 (2012); M. Pieri (on behalf of the CMS Collaboration), arXiv:1205.2907.

[3] G. Davies et al. (Tevatron New Phenomena and Higgs Working Group, CDF Collaboration, and D0 Collaboration), arXiv:1203.3774.

[4] R. Barate et al. (ALEPH Collaboration, DELPHI Collaboration, L3 Collaboration, OPAL Collaboration, and LEP Working Group for Higgs Boson Searches) Phys. Lett. B 565, 61 (2003).

[5] G. Aad et al. (ATLAS Collaboration), Phys. Lett. B 705, 452 (2011); Phys. Rev. Lett. 108, 111803 (2012).

[6] S. Chatrchyan et al. (CMS Collaboration), Phys. Lett. B 710, 403 (2012).

[7] G. Aad et al. (ATLAS Collaboration), Phys. Lett. B 705, 435 (2011); 710, 383 (2012).

[8] S. Chatrchyan et al. (CMS Collaboration), Phys. Rev. Lett. 108, 111804 (2012).
[9] G. Aad et al. (ATLAS Collaboration), Phys. Rev. Lett. 108, 111802 (2012); (), Phys. Lett. B 716, 62 (2012).

[10] S. Chatrchyan et al. (CMS Collaboration), Phys. Lett. B 699, 25 (2011); 710, 91 (2012).

[11] S. Chatrchyan et al. (CMS Collaboration), Phys. Lett. B 713, 68 (2012).

[12] G. Aad et al. (ATLAS Collaboration), arXiv:1206.5971.

[13] S. Chatrchyan et al. (CMS Collaboration), Phys. Lett. B 710, 284 (2012).

[14] G. Aad et al. (ATLAS Collaboration), arXiv:1207.0210.

[15] B. A. Kniehl, Phys. Rep. 240, 211 (1994).

[16] M. Spira, Fortschr. Phys. 46, 203 (1998); B. A. Kniehl, Int. J. Mod. Phys. A 17, 1457 (2002); A. Djouadi, Phys. Rep. 457, 1 (2008); J. Ellis, M. K. Gaillard, and D. V. Nanopoulos, arXiv:1201.6045.

[17] J. R. Ellis, M. K. Gaillard, and D. V. Nanopoulos, Nucl. Phys. B106, 292 (1976); B. L. Ioffe and V. A. Khoze, Fiz. Elem. Chastits At. Yadra 9, 118 (1978) [Sov. J. Part. Nucl. 9, 50 (1978)]; A. I. Vaŭnshteĭn, M. B. Voloshin, V. I. Zakharov, and M. A. Shifman, Yad. Fiz. 30, 1368 (1979) [Sov. J. Nucl. Phys. 30, 711 (1979)]. 
[18] H.-Q. Zheng and D.-D. Wu, Phys. Rev. D 42, 3760 (1990); A. Djouadi, M. Spira, J. J. van der Bij, and P. M. Zerwas, Phys. Lett. B 257, 187 (1991); S. Dawson and R.P. Kauffman, Phys. Rev. D 47, 1264 (1993); A. Djouadi, M. Spira, and P. M. Zerwas, Phys. Lett. B 311, 255 (1993); K. Melnikov and O. I. Yakovlev, Phys. Lett. B 312, 179 (1993); M. Inoue, R. Najima, T. Oka, and J. Saito, Mod. Phys. Lett. A 9, 1189 (1994); J. Fleischer, O. V. Tarasov, and V. O. Tarasov, Phys. Lett. B 584, 294 (2004); R. Harlander and P. Kant, J. High Energy Phys. 12 (2005) 015.

[19] M. Steinhauser, in Proceedings of the Ringberg Workshop on the Higgs Puzzle-What Can We Learn from LEP2, LHC, NLC, and FMC?, Ringberg Castle, Germany, 8-13 December 1996, edited by B. A. Kniehl (World Scientific, Singapore, 1997), p. 177; K. G. Chetyrkin, B. A. Kniehl, and M. Steinhauser, Nucl. Phys. B510, 61 (1998).

[20] U. Aglietti, R. Bonciani, G. Degrassi, and A. Vicini, Phys. Lett. B 595, 432 (2004).

[21] A. Djouadi, P. Gambino, and B. A. Kniehl, Nucl. Phys. B523, 17 (1998).

[22] F. Fugel, B. A. Kniehl, and M. Steinhauser, Nucl. Phys. B702, 333 (2004); F. Fugel, Acta Phys. Pol. B 38, 761 (2007); J. Brod, F. Fugel, and B. A. Kniehl, Phys. Rev. D 78, 011303(R) (2008); Nucl. Phys. B807, 188 (2009).

[23] G. Degrassi and F. Maltoni, Nucl. Phys. B724, 183 (2005); G. Passarino, C. Sturm, and S. Uccirati, Phys. Lett. B 655 , 298 (2007).

[24] E. Braaten and J. P. Leveille, Phys. Rev. D 22, 715 (1980); N. Sakai, Phys. Rev. D 22, 2220 (1980); T. Inami and T. Kubota, Nucl. Phys. B179, 171 (1981); M. Drees and K.-I. Hikasa, Phys. Lett. B 240, 455 (1990); 262, 497(E) (1991).

[25] J. Fleischer and F. Jegerlehner, Phys. Rev. D 23, 2001 (1981).

[26] D. Yu. Bardin, B. M. Vilenskiŭ, and P. Kh. Khristova, Yad. Fiz. 53, 240 (1991) [Sov. J. Nucl. Phys. 53, 152 (1991)]; B. A. Kniehl, Nucl. Phys. B376, 3 (1992); A. Dabelstein and W. Hollik, Z. Phys. C 53, 507 (1992).

[27] S. G. Gorishny, A. L. Kataev, S. A. Larin, and L. R. Surguladze, Mod. Phys. Lett. A 5, 2703 (1990); Phys. Rev. D 43, 1633 (1991); A. L. Kataev and V. T. Kim, Mod. Phys. Lett. A 9, 1309 (1994).

[28] L. R. Surguladze, Phys. Lett. B 341, 60 (1994).

[29] B. A. Kniehl, Phys. Lett. B 343, 299 (1995).

[30] K. G. Chetyrkin and A. Kwiatkowski, Nucl. Phys. B461, 3 (1996).

[31] A. L. Kataev, Pis'ma Zh. Éksp. Teor. Fiz. 66, 308 (1997); [, JETP Lett. 66, 327 (1997)].

[32] B. A. Kniehl and A. Sirlin, Phys. Lett. B 318, 367 (1993); B. A. Kniehl, Phys. Rev. D 50, 3314 (1994); A. Djouadi and P. Gambino, Phys. Rev. D 51, 218 (1995); 53, 4111(E) (1996).

[33] A. Kwiatkowski and M. Steinhauser, Phys. Lett. B 338, 66 (1994); 342, 455(E) (1995); B. A. Kniehl and M. Spira, Nucl. Phys. B432, 39 (1994); B. A. Kniehl, Int. J. Mod. Phys. A 10, 443 (1995).

[34] M. Butenschön, F. Fugel, and B. A. Kniehl, Phys. Rev. Lett. 98, 071602 (2007); Nucl. Phys. B772, 25 (2007).

[35] L. Durand, B. A. Kniehl, and K. Riesselmann, Phys. Rev. Lett. 72, 2534 (1994); 74, 1699(E) (1995); Phys. Rev. D 51, 5007 (1995); A. Ghinculov, Phys. Lett. B 337, 137 (1994); 346, 426(E) (1995); V. Borodulin and G. Jikia, Phys. Lett. B 391, 434 (1997).
[36] K. G. Chetyrkin, Phys. Lett. B 390, 309 (1997).

[37] K. G. Chetyrkin and M. Steinhauser, Phys. Lett. B 408, 320 (1997).

[38] B. A. Kniehl and M. Steinhauser, Nucl. Phys. B454, 485 (1995); Phys. Lett. B 365, 297 (1996).

[39] K. G. Chetyrkin, B.A. Kniehl, and M. Steinhauser, Phys. Rev. Lett. 78, 594 (1997); Nucl. Phys. B490, 19 (1997).

[40] P. A. Baikov, K. G. Chetyrkin, and J. H. Kühn, Phys. Rev. Lett. 96, 012003 (2006).

[41] A. L. Kataev and V.T. Kim, Proc. Sci., ACAT08 (2008) 004, arXiv:0902.1442.

[42] B. A. Kniehl, Phys. Lett. B 244, 537 (1990); A. Grau, G. Panchieri, and R. J. N. Phillips, Phys. Lett. B 251, 293 (1990).

[43] B. A. Kniehl, Nucl. Phys. B357, 439 (1991).

[44] B. A. Kniehl, Nucl. Phys. B352, 1 (1991).

[45] D. Yu. Bardin, B. M. Vilenskiı̌, and P. Kh. Khristova, Yad. Fiz. 54, 1366 (1991) [Sov. J. Nucl. Phys. 54, 833 (1991)].

[46] A. Bredenstein, A. Denner, S. Dittmaier, and M. M. Weber, Phys. Rev. D 74, 013004 (2006); J. High Energy Phys. 02 (2007) 080.

[47] B. A. Kniehl, Phys. Rev. D 53, 6477 (1996).

[48] B. A. Kniehl and M. Spira, Nucl. Phys. B443, 37 (1995); Z. Phys. C 69, 77 (1995).

[49] A. Ghinculov, Nucl. Phys. B455, 21 (1995); A. Frink, B. A. Kniehl, D. Kreimer, and K. Riesselmann, Phys. Rev. D 54, 4548 (1996).

[50] B. A. Kniehl, Z. Phys. C 55, 605 (1992).

[51] A. Denner, J. Küblbeck, R. Mertig, and M. Böhm, Z. Phys. C 56, 261 (1992); A. Denner, B. A. Kniehl, and J. Küblbeck, DESY Report No. 92-123A, 1992, p. 31.

[52] S. Chatrchyan et al. (CMS Collaboration), J. High Energy Phys. 03 (2012) 081.

[53] B. A. Kniehl, Phys. Lett. B 282, 249 (1992).

[54] G. Póksik and T. Torma, Z. Phys. C 6, 1 (1980); T. G. Rizzo, Phys. Rev. D 22, 722 (1980).

[55] W.-Y. Keung and W. J. Marciano, Phys. Rev. D 30, 248(R) (1984).

[56] B. A. Kniehl, Comput. Phys. Commun. 58, 293 (1990).

[57] B. A. Kniehl, Nucl. Phys. B347, 86 (1990).

[58] B. A. Kniehl and A. Sirlin, Nucl. Phys. B371, 141 (1992).

[59] M. Consoli, W. Hollik, and F. Jegerlehner, in Z Physics at LEP 1, edited by G. Altarelli, R. Kleiss, and C. Verzegnassi (1989), Vol. 1, p. 7 (CERN Yellow Report No. 89-08); W. F. L. Hollik, Fortschr. Phys. 38, 165 (1990); F. Halzen, B. A. Kniehl, and M. L. Stong, in Particle Physics: VI Jorge André Swieca Summer School, Campos de Jordão, Brasil, 14-26 January, 1991, edited by O. J. P. Éboli, M. Gomes, and A. Santoro (World Scientific, Singapore, 1992), p. 219; Z. Phys. C 58, 119 (1993).

[60] S. Dawson and S. Willenbrock, Phys. Lett. B 211, 200 (1988).

[61] M. Veltman, Nucl. Phys. B123, 89 (1977); M. B. Einhorn, D. R. T. Jones, and M. Veltman, Nucl. Phys. B191, 146 (1981).

[62] J. J. van der Bij and F. Hoogeveen, Nucl. Phys. B283, 477 (1987); M. Consoli, W. Hollik, and F. Jegerlehner, Phys. Lett. B 227, 167 (1989). 
[63] A. Djouadi and C. Verzegnassi, Phys. Lett. B 195, 265 (1987); A. Djouadi, Nuovo Cimento A 100, 357 (1988); B. A. Kniehl, J. H. Kühn, and R. G. Stuart, Phys. Lett. B 214, 621 (1988).

[64] L. Avdeev, J. Fleischer, S. Mikhailov, and O. Tarasov, Phys. Lett. B 336, 560 (1994); 349, 597(E) (1995); K. G. Chetyrkin, J. H. Kühn, and M. Steinhauser, Phys. Lett. B 351, 331 (1995).
[65] J. Beringer et al. (Particle Data Group Collaboration), Phys. Rev. D 86, 010001 (2012).

[66] K. G. Chetyrkin, B. A. Kniehl, and M. Steinhauser, Phys. Rev. Lett. 79, 2184 (1997); Y. Schröder and M. Steinhauser, J. High Energy Phys. 01 (2006) 051; K. G. Chetyrkin, J. H. Kühn, and C. Sturm, Nucl. Phys. B744, 121 (2006); B. A. Kniehl, A. V. Kotikov, A. I. Onishchenko, and O. L. Veretin, Phys. Rev. Lett. 97, 042001 (2006). 